\title{
Implementation of healthy lifestyle promotion in primary care: Patients as coproducers
}

\author{
Kristin Thomas, Preben Bendtsen and Barbro Krevers
}

Linköping University Post Print

Tweet

N.B.: When citing this work, cite the original article.

Original Publication:

Kristin Thomas, Preben Bendtsen and Barbro Krevers, Implementation of healthy lifestyle promotion in primary care: Patients as coproducers, 2014, Patient Education and Counseling, (97), 2, 283-290.

http://dx.doi.org/10.1016/j.pec.2014.07.033

Copyright: Elsevier

http://www.elsevier.com/

Postprint available at: Linköping University Electronic Press

http://urn.kb.se/resolve?urn=urn:nbn:se:liu:diva-112827 


\section{Implementation of healthy lifestyle promotion in primary care:}

\section{patients as coproducers}

Kristin Thomas $^{\mathrm{a}, *}$, Preben Bendtsen ${ }^{\mathrm{a}, \mathrm{c}}$, Barbro Krevers ${ }^{\mathrm{b}}$

${ }^{a}$ Department of Medical and Health Sciences, Division of Community Medicine, Linköping

University, 58183 Linköping, Sweden

${ }^{b}$ Department of Medical and Health Sciences, Division of Health Care Analysis, Linköping

University, Linköping, Sweden

${ }^{c}$ Department of Medical Specialist and Department of Medical and Health Sciences, Linköping

University, Motala, Sweden

*Corresponding author: Tel.: +46 10103 2385; fax: +46 10103 1865. E-mail address:

kristin.thomas@liu.se (K. Thomas). 


\begin{abstract}
Objectives: To explore and theorize how patients perceive, interpret, and react in health behavior promotion situations in primary care and to investigate patients' role in implementation of lifestyle promotion illustrated by typologies.

Methods: Grounded theory was used to assess qualitative interview data from 22 patients with varied experience of healthy lifestyle promotion. Data were analyzed by constant comparative analysis.

Results: A substantive theory of being healthy emerged from the data. The theory highlights the processes that are important for implementation before, during, and after lifestyle promotion. Three interconnected categories emerged from the data: conditions for being healthy, managing being healthy, and interactions about being healthy; these formed the core category: being healthy. A typology proposed four patient trajectories on being healthy: resigned, receivers, coworkers, and leaders.

Conclusion: Patients coproduced the implementation of lifestyle promotion through the degree of transparency, which was a result of patients' expectations and situation appraisals.

Practice implications: Different approaches are needed during lifestyle promotion depending on a variety of patient-related factors. The typology could guide practitioners in their lifestyle promotion practice.
\end{abstract}

Keywords: Implementation; Primary care; Lifestyle promotion; Grounded theory 


\section{Introduction}

The implementation of healthy lifestyle promotion in primary care has not been optimal, i.e., the promotion of physical activity, tobacco cessation, a balanced diet, and moderate alcohol intake. Rates of lifestyle promotion in primary care vary internationally from a few percent to about 50\% [1-4]. However, similar to other European countries, almost every second Swede engages in at least one health-compromising behavior and would potentially benefit from lifestyle promotion $[5,6]$.

The challenge in implementing lifestyle promotion has mainly been explained by contextual factors inherent in the organization, practitioner-related factors, and attributes of practice methods [7-10]. Furthermore, barriers to implementing lifestyle promotion include willingness of the clinician, lack of time, and lack of training and knowledge [11-14]. Interventions to facilitate implementation have therefore primarily focused on altering clinical behavior, redesigning organization systems, or generating implementation guidelines [15-20]. Practitioner-level interventions, e.g., group education, reminders, or multifaceted interventions, have been shown to have a small to moderate effect on implementation. The evidence underlying organizational interventions, e.g., revised computer systems, has been limited $[17,19]$.

However, depending on how successful implementation is defined, different factors are deemed important to investigate. If implementation is defined as simply giving lifestyle advice, patients become receivers and their role in the implementation process is less important. However, if implementation is defined as the exchange of information and strategies for lifestyle change, patients become a vital stakeholder in the implementation process. Indeed, there are studies indicating that patients indirectly and directly influence the implementation of lifestyle promotion. 
For example, fear of offending or being perceived as judgmental has been found to hinder practitioners from bringing up lifestyle with patients [21,22]. Patients' attitudes and behaviors regarding healthy living have been found to influence the implementation of lifestyle promotion $[12,23,24]$. For example, patients who explicitly express readiness to change are more likely to receive advice and recommendations regarding healthy living [22]. Furthermore, patients have been reported to initiate discussions about healthy living thus influencing implementation directly [21-23]. Also, patients differ in their preferences on lifestyle promotion, e.g., level of support and information, which may influence how lifestyle promotion is implemented [25]. However, there is a knowledge gap on the mechanisms whereby patients influence the implementation process of lifestyle promotion.

This study aimed to explore and theorize on how patients perceive, interpret, and react in healthy lifestyle promotion situations in primary care. Built on a patient perspective, the aim was also to investigate patients' role in implementation of lifestyle promotion illustrated by typologies. Findings could improve future efforts to implement lifestyle promotion in primary care. 


\section{Methodology}

Grounded theory [26] was chosen using qualitative interview data. Grounded theory stresses rigor throughout the research process, from study design to data collection and analysis, resulting in the generation of a substantive theory. By using grounded theory, social processes and interactions between patients and practitioners can be explored from a patient perspective.

\subsection{Informants and setting}

Informants were recruited from six primary care centers located in eastern Sweden, a region with approximately 411000 inhabitants. Purposive sampling was used to generate a participant pool. Invitations were posted to patients with information and details on the confidentiality of the research project. Informants expressed their interest by telephone, e-mail, or using the reply slip attached to the invitation. The criterion for participation was the ability to speak and understand Swedish.

There were two stages of recruitment. The first stage aimed to recruit patients who had experienced extended lifestyle promotion, i.e., counselling. It was thought that these patients would offer rich data on the lifestyle promotion situation. Primary care registers of patient visits were used to identify these patients. Invitations were sent out to all patients $(n=42)$ who had experienced extended lifestyle promotion in the previous 4 weeks. Six informants (3 women and 3 men) showed an interest and were interviewed. The second recruitment stage aimed to maximize the theoretical scope by recruiting informants with varied experience of lifestyle promotion. A random selection of 250 registered patients of varied age and gender were sent invitations; 33 (23 women and 10 men) showed interest. The first author contacted everybody who was interested in taking part to provide information about the study and to gather descriptive data: age, gender, occupation, education, and experience of lifestyle promotion. Eight ( 4 women and 4 men) opted out or could not be reached. Sixteen (12 women and four men) were selected for interview all of whom took part. The descriptive data and 
initial contact were used for theoretical sampling, i.e., to recruit informants who could provide data that advanced the analysis, in accordance with grounded theory [26]. After 20 interviews, two subsequent interviews did not offer any new data, which suggested that theoretical saturation had been reached.

Totally, twenty-two informants (15 women and 7 men) took part in interviews. Ages ranged from 30 to 78 years. Informants included individuals with varied lifestyles and experience of lifestyle promotion, as well as individuals who had initiated lifestyle promotion themselves and those that had not; the characteristics of the informants are presented in Table 1.

\subsection{Data collection}

Interviews and data analysis were carried out from May 2012 to September 2013 by KT. Informants could choose interview location and mode, i.e., face to face $(n=12)$ or by telephone $(n=10)$. The informants were asked to speak freely about two topics: experience of lifestyle change and experience of healthy lifestyle promotion in primary care. Sub-questions were prepared and used as prompts if needed, e.g., "how did it feel to talk about your lifestyle?" However, the sub-questions changed during data collection and analyses, as categories emerged and were explored further in subsequent interviews. The interview guide was continuously adapted in accordance with grounded theory [26]. Interviews were audiorecorded and transcribed verbatim and lasted between 40 and 75 minutes. Thoughts and perceptions that had surfaced during the interviews were recorded in field memos, which complemented the interview transcripts. Data collection and analysis were intertwined.

\subsection{Data analysis}

Research techniques such as open coding, constant comparison, memos and theoretical coding were used in accordance with grounded theory [26]. Transcribed interviews were initially coded (using open coding) line by line. Indicators and incidents relevant to the aim 
were grouped together to form categories. During a continuous analysis process, categories that had emerged were either confirmed or modified, and new categories were identified. Analysis of how categories related to each other was carried out using theoretical coding. Throughout the process, constant comparison was used between and within transcripts, codes, and categories. Analysis of the data guided further data collection by exploring identified codes and categories in subsequent interviews. Memos were used throughout the data collection and analysis to record observations, thoughts, ideas, interpretations, hypotheses and questions. Theoretical memos were used during the analysis as worksheets to keep track of ideas and thoughts during the analysis. Data analysis was predominantly a collaborative effort between $\mathrm{KT}$ and BK. A progressive analysis process was used whereby KT carried out the initial analysis, which was then discussed by KT and BK. Subsequently, KT carried out more interviews and analysis, which was followed by more discussions between KT and BK, and so forth. Preliminary analyses were presented to PB and were discussed recurrently with all authors. In the case of disagreement, the authors revisited the data and aimed to reach consensus after further discussions.

\subsection{Ethical considerations}

The research project received the approval of the local Central Ethical Review Board (DNR: IMH-2009-00335). 


\section{Results}

A substantive theory of being healthy emerged from data including three interconnected categories: conditions for being healthy, managing being healthy, and interactions on being healthy; these formed the core category: being healthy (Fig. 1). Two of the categories (conditions for being healthy and managing being healthy) were continuous processes that changed over time. Lifestyle promotion, or interactions on being healthy, could be a single or recurring event. The categories occurred in three phases all relevant to the implementation process: before, during, and after meeting with practitioners. A typology was generated including four patient types: resigned, receivers, coworkers, and leaders (Fig. 2). The processes for these types differed before, during, and after lifestyle promotion, and ultimately how being healthy was approached.

\subsection{Being healthy}

The core category, being healthy, refers to the process by which patients approached their health ideals. Being healthy included behavioral, emotional, cognitive, and pragmatic changes, all with the purpose of advancing toward a particular health ideal. For example, being physically active to reach an ideal weight; adopting a healthy diet to prevent high cholesterol; or making changes to reduce stress levels.

Being healthy co-occurred with the process of maximizing well-being. Being healthy was a process to reach a future health goal, whereas well-being was a state of mind in the present. Well-being included physical, mental, financial, spiritual, existential, social, and emotional aspects. A dilemma occurred among patients when there was a dispute between acts of being healthy and acts leading to well-being, e.g., stop smoking to be healthy versus smoking a cigarette to feel good. Patients resolved this in different ways, e.g., choosing wellbeing, choosing being healthy, or trying to balance the two. Well-being could be maximized with or without being healthy. Thus, in the process of approaching health ideals, through being healthy, sometimes incompatibilities with well-being had to be considered. The balance 
between the two was characterized by the patients' conditions for being healthy and how they managed being healthy.

\subsubsection{Conditions for being healthy}

Conditions for being healthy comprised three subcategories: patients' conceptions, drive, and resources regarding being healthy. The patients' unique set of conditions contributed to their expectations of the lifestyle promotion situation and interactions about being healthy.

Conceptions included definitions and attribution of responsibility, e.g., what does being healthy entail and who is responsible for me being healthy. Conceptions were individual and generated from knowledge about health, previous experiences, and influences from media and social networks, e.g., health service, workplace, or family.

\footnotetext{
... to try to change is one thing but deciding to change is another... then it was finished [smoking] .. once I decided I became more stubborn ... it is up to the individual although you can get tips and advice.
}

Drives for being healthy were perceived to be essential to prevent illness or promote health and were internal or external. External drives could be prompts from family, a diagnosis, test results, or other tangible health risks. Internal drives could be recognition of needs and benefits or enjoyment and interest in being healthy.

\footnotetext{
Now it is the risk for heart disease or something similar that you try to ... minimize the risk for some time in the future.
}

Resources for being healthy were also internal or external. Internal resources included well-being and feeling resourceful, which led to a capacity for being healthy in the long term. External resources included social support and welfare in life areas, e.g., financial stability, rewarding employment, or significant others keeping well. Instability in life areas made being healthy difficult because external resources become scarce and these life areas would in turn require support.

To have a job and feel well with family and friends around ... if you don't have that, it is difficult to stay healthy. 


\subsubsection{Managing being healthy}

During managing being healthy, patients tried to make changes that would bring them closer to their health ideals while maximizing well-being. Two subcategories emerged: embracing and evading being healthy. The processes occurred simultaneously and are each a continuum.

Patients with optimal conditions primarily engaged in embracing being healthy. Optimal conditions included internal drives and resources for being healthy. These patients successfully incorporated being healthy with well-being using more or less conscious regimens. Embracing could be pragmatic (e.g., reducing barriers or making weekly shopping lists), behavioral (e.g., substituting juice with water), emotional (e.g., internal pep talk), or cognitive (e.g., goal setting). Lifestyle promotion predominantly offered patients support that facilitated pragmatic, behavioral, and cognitive regimens.

... there is always one that sits on your shoulder and says 'take some more'; 'no' says the other one; then you have to choose which one is the strongest.

Patients with suboptimal conditions primarily engaged in evading being healthy. Suboptimal conditions included limited internal drives and resources for being healthy. These patients often chose well-being rather than being healthy. Several ways of evading emerged: avoiding uncomfortable or unfamiliar situations; forgetting when and where change was intended, leading to the adoption of change fizzling out; compromising, i.e., patients allowed themselves to take short cuts or cheat; and rebelling, when patients behaved in opposition to recommendations. Compromising and rebelling were conscious ways of evading. These were examples where interactions about being healthy could contribute to unhelpful ways of managing being healthy. Both could be direct responses of content or treatment. Compromising was also apparent with patients who attributed internal responsibility for being healthy but had scarce resources for being healthy.

I have all the tools. I know what to do and what I can handle, but even so, if I know that nobody is checking on me, I take shortcuts. 


\subsubsection{Interactions about being healthy}

Interactions about being healthy consisted of patients sharing information (past experiences, current situation, and future needs) and handling the information they received (test results, recommendations, or advice). Patients did this with a varied degree of transparency depending on their expectations and appraisal of the situation.

Patients' expectations (implicit or explicit) were generated from patients' conditions for being healthy, and previous experience of managing being healthy and lifestyle promotion events. Expectations referred to their role as patients (e.g., responsibilities and obligations); the practitioner's role (e.g., which profession to meet, communication style, personality qualities) and the consultation (purpose, content and care preferences).

Patients continuously appraised the situation based on their expectations and accommodated their interactions accordingly. For example if the practitioner was perceived as trustworthy patients felt more comfortable and were transparent in how they shared and handled the information. Contrary, if the situation was appraised as negative, patients could become less transparent and, e.g., did voice worries or disagreements.

Thus, there was a continuous interaction between patients' expectations, appraisal and degree of transparency, e.g., can I trust this individual or is this relevant information for this meeting. High transparency was characterized by honesty and openness; low transparency by camouflaging and censoring. Low degree of transparency could result in that lifestyle promotion was less tailored to patients' preferences. Subsequently, these patients were less equipped to embrace being healthy.

I told her that I had been more active even though I hadn't because I thought she might get angry otherwise.

I mean you open up and we talk about everything, you have to open up and share what the problem is. 


\subsection{Typology of how patients approached being healthy}

A typology of patient trajectories was generated based on the patients' characteristics regarding the categories. The typology illustrates how the categories are interconnected and occur in three phases, all of which are relevant for the implementation of lifestyle promotion. The typologies are not constant, i.e., one individual could be one type at one point and another in the next (Fig. 2).

Four patient types emerged from the data: resigned, receivers, coworkers, and leaders. These types were predominantly passive or active during lifestyle promotion. Passive patient types attributed external responsibility to being healthy, had predominantly external drives and resources for being healthy, and had challenging experiences of managing being healthy. During lifestyle promotion, passive types listened and gave short answers to questions. Active patient types attributed internal responsibility for being healthy, had internal and external drives and resources for being healthy, and had largely positive experiences of managing being healthy. During lifestyle promotion, active types would injected new angles on topics, asked questions, and gave comprehensive answers.

\subsubsection{Resigned}

Resigned type predominantly attributed external responsibility to being healthy. This type had no or scarce internal resources for being healthy. Thus, the resigned type depended on external support and had no or predominantly external drives for being healthy. This type resorted to evading rather than embracing being healthy before lifestyle promotion. Resigned type adopted a predominantly passive role during lifestyle promotion where a positive situation appraisal was central to a high degree of transparency. Positive situation appraisal together with a high degree of transparency was essential for embracing being healthy after promotion (Fig. 2a). 


\subsubsection{Receivers}

Receivers predominantly attributed internal responsibility to being healthy. They had scarce internal resources and drives for being healthy, which made it difficult to manage being healthy. However, this type had external resources for being healthy that could act as reminders for change. Receivers adopted a predominantly passive role during promotion and a positive situation appraisal was important for a high degree of transparency. Positive situation appraisal was not necessarily sufficient for a high degree of transparency. Despite showing an interest in receiving information and asking questions, transparency could be limited. Positive situation appraisal together with a high degree of transparency contributed to embracing being healthy after experiencing lifestyle promotion (Fig. 2b).

\subsubsection{Coworkers}

Coworkers mainly attributed internal responsibility to being healthy. This type had both internal and external drives for being healthy. They also had some internal resources that made them less dependent on external resources. This type engaged more in embracing than evading being healthy before lifestyle promotion. Coworkers expected to have a predominantly active role and worked together with practitioners to reach a realistic solution or plan for being healthy. Accordingly, information sharing and handling were mostly transparent. Negative situation appraisal could lead to either a low or high degree of transparency. The higher the degree of transparency, the more embracing being healthy was facilitated (Fig. 2c).

\subsubsection{Leaders}

Leaders attributed internal responsibility to being healthy. This type had both internal resources and drives that led to goal orientation and less dependency on external resources. Leaders expected to have an active role by sometimes taking the lead in conversations. Leaders perceived themselves to be the primary stakeholder in the lifestyle promotion session and accordingly shared and handled information transparently. This type predominantly engaged in embracing being healthy before and after lifestyle promotion. Positive situation appraisal or a 
high degree of transparency was not important for this patient type to embrace being healthy after experiencing lifestyle promotion (Fig. 2d).

\section{Discussion and conclusion}

This study aimed to explore and theorize how patients perceive, interpret, and react in lifestyle promotion situations in primary care. Three interconnected categories emerged from the data: conditions for being healthy, interactions about being healthy, and managing being healthy. The categories form a substantive theory of being healthy. The theory highlights the processes that are important for implementation before, during, and after lifestyle promotion. A typology of patient trajectories proposed four patient types: resigned, receivers, coworkers, and leaders. The typology illustrates how the categories are interconnected and how patient types differ across categories leading to different lifestyle promotion outcomes.

\subsection{Discussion}

\subsubsection{Theory of being healthy}

The theory of being healthy echoes prevalent theories about motivation and behavior change. Specifically, the categories managing and conditions for being healthy support other theories by highlighting both external and internal factors for staying healthy. For example, SDT proposes that varied extrinsic and intrinsic sources of motivation direct behaviors [27]. Furthermore, COM-B suggests that an individual's capability, opportunity (physical environment) and motivation are important for future behavior [28]. By using a patient and process perspective, the theory of being healthy, contributes to how we understand lifestyle change and lifestyle promotion, and how these two interact in a process of being healthy.

Indeed, the patient typology illustrates how processes before, during, and after lifestyle promotion are interconnected. For example, patient types with suboptimal conditions before experiencing lifestyle promotion were more vulnerable to negative experiences during lifestyle promotion (e.g., not feeling listened to). In turn, negative experiences during lifestyle 
promotion could further worsen the conditions for change after lifestyle promotion. Thus, the findings bring together aspects of motivation and lifestyle behavior change within the implementation situation in an important way.

\subsubsection{Patients' role in implementation}

The findings suggest that patients coproduced the implementation of lifestyle promotion in an active or passive way. Patients appraised the situation, reacted, and acted in their interactions with practitioners. Specifically, through the degree of transparency, patients can steer lifestyle promotion. Research on patients' role in health care delivery have transformed from patient compliance (i.e., patients following the recommended treatment) to concordance whereby patients and practitioners are perceived as equals and the patients' role in care decision making is acknowledged $[29,30]$. Implementation theory could be enriched by adopting a similar approach that would include perceiving patients as active or passive agents of implementation. This could be particularly important for the area of lifestyle promotion. For promotion to have an impact on health outcomes a commitment is required from patients, e.g., coproducing realistic plans for behavior change. Thus, prevalent understanding of the implementation of lifestyle promotion in health care could be improved if patients were seen as coproducers rather than receivers.

Recent implementation theory proposes that both patient and practitioner acceptability are important implementation outcomes. Acceptability refers to the perceptions among stakeholders that a treatment is agreeable. Acceptability changes over time according to experience and differs between treatments. Lack of acceptability challenges implementation [31]. The degree of transparency in the current findings may be an indicator of patient acceptability of lifestyle promotion illustrated by how patients engaged with and welcomed advice. This would suggest that patient acceptability is a result of expectations before and appraisal during lifestyle promotion. Furthermore, that patient acceptability can be increased or decreased during promotion depending on patients' appraisal. 


\subsubsection{Methodological strengths and limitations}

Applying a patient perspective is a potential strength of the study. Previous research on the implementation of lifestyle promotion has mainly focused on practitioner perceptions, thus the current findings add to this research. Furthermore, the age range of the informants (30-78 years) was broad, and thus captured the target group for lifestyle promotion. Only 7 men compared with 15 women took part in the interviews. However, the findings did not suggest that the phenomena emerging from the data differed due to age or gender.

\subsection{Conclusions}

A substantive theory of being healthy emerged from the data. The theory highlights processes that are important for implementation before, during, and after the lifestyle promotion event. Patients coproduced the implementation of lifestyle promotion through the degree of transparency, which was a result of patients' expectations and situation appraisals. The typology of patient trajectories suggested that different approaches are needed during lifestyle promotion depending on a variety of patient-related factors. The typology could guide practitioners in their lifestyle promotion practice.

\subsection{Practice implications}

Barriers for implementing lifestyle promotion have included insufficient training and worry about patient reactions [21-23]. The findings in this study indicated that there were individual differences in patient expectations, affirming practitioners' concerns for caution. However, when lifestyle promotion coexisted with positive practitioner characteristics (e.g., trust and empathy), negative expectations were revalued. Thus, the findings may reassure practitioners and confirm the importance of a trustful patient-practitioner interaction, which is described repeatedly in the literature $[18,32,33]$.

The typology suggests that different approaches are needed during lifestyle promotion depending on a variety of patient-related factors before the promotion. Emotional support, trust, 
and empathy are more important for patient types with limited conditions before promotion, whereas brief advice suffices for other types. This is consistent with patient-centered care; however, the current study further illustrates the complexity of the issue [34]. Patient types with scarce conditions were more vulnerable to negative experiences during lifestyle promotion (e.g., unfulfilled expectations or treatment). These patient types often responded with destructive strategies (e.g., rebelling against practitioners' recommendations). Thus, failing to identify the most suitable approach could not only result in insufficient support but potentially leave patients worse off than before the promotion. This suggests that lifestyle promotion is an immense task requiring specialist competency, including the ability to identify the best approach for individual patients; this process requires taking patients' conditions for being healthy and managing change into account. However, the conditions in current primary care practice (e.g., time and resources) for implementing this type of practice may be limited. The typology could support and guide practitioners in identifying the best approaches for individual patients. The next step is to generate a screening tool based on patient typologies so that patients' conditions and experiences of being healthy are identified. For example, a screening tool could include questions to identify patients' conditions and experiences of managing being healthy before lifestyle promotion in order to tailor the advice and communication style. Furthermore, reorganization solutions in primary care (e.g., coordinated care) could reduce the barriers to the practice of lifestyle promotion through access to specialized competency $[35,36]$.

The authors confirm all patient identifiers have been removed or disguised so the patient described are not identifiable and cannot be identified through the details of the story.

\section{Acknowledgements}

The study was funded by Östergötland County Council and Linköping University. We thank the participating primary care centres for providing access to conduct the study. 


\section{References}

[1] Manuti B, Rizza P, Bianco A, Nobile CGA, Pavia M. The quality of preventive health care delivered to adults : results from a cross-sectional study in Southern Italy. BMC Public Health 2010;10:350.

[2] Noordman J, Verhaak P, van Dulmen S. Discussing patient's lifestyle choices in the consulting room: analysis of GP-patient consultations between 1975 and 2008. BMC Fam Pract 2010;11:87.

[3] Brotons C, Lobos JM, Royo-Bordonada MÁ, Maiques A, de Santiago A, Castellanos Á, et al. Implementation of Spanish adaptation of the European guidelines on cardiovascular disease prevention in primary care. BMC Fam Pract 2013;14:36.

[4] Swedish Association of Local Authorities and Region. National Patient Survey. Http://npe.skl.se/ 2012.

[5] National Board of Health and Welfare. Tobacco, alcohol, physical activity and eating habits. 2012.

[6] World Health Organisation. Noncommunicable Diseases: Country Profiles. 2011.

[7] Bhattacharyya O, Reeves S, Zwarenstein M. What Is Implementation Research?: Rationale, Concepts, and Practices. Res Soc Work Pract 2009;19:491-502.

[8] Greenhalgh T, Robert G, Macfarlane F, Bate P, Kyriakidou O. Diffusion of Innovations in Service Organizations: Systematic Review and Recommendations. Innov Serv Organ 2004;82:581-629.

[9] Rogers EM. Diffusion of preventive innovations. Addict Behav 2002;27:989-93.

[10] Damschroder LJ, Aron DC, Keith RE, Kirsh SR, Alexander J a, Lowery JC. Fostering implementation of health services research findings into practice: a consolidated framework for advancing implementation science. Implement Sci 2009;4:50.

[11] Brotons C, Björkelund C, Bulc M, Ciurana R, Godycki-Cwirko M, Jurgova E, et al. Prevention and health promotion in clinical practice: the views of general practitioners in Europe. Prev Med (Baltim) 2005;40:595-601.

[12] Lambe B, Collins C. A qualitative study of lifestyle counselling in general practice in Ireland. Fam Pract 2010;27:219-23.

[13] Saliba M, Sammut MR, Vickers KS, Calleja N. Health behaviour counselling in primary care : general practitioner - reported rate and confidence. Malta Med J 2011;23:22-8.

[14] Calderón C, Balagué L, Cortada JM, Sánchez A. Health promotion in primary care: how should we intervene? A qualitative study involving both physicians and patients. BMC Health Serv Res 2011;11:62.

[15] Aspy CB, Mold JW, Thompson DM, Blondell RD, Landers PS, Reilly KE, et al. Integrating screening and interventions for unhealthy behaviors into primary care practices. Am J Prev Med 2008;35:S373-80. 
[16] Ben-Arye E, Lear A, Hermoni D, Margalit RS. Promoting lifestyle self-awareness among the medical team by the use of an integrated teaching approach: a primary care experience. J Altern Complement Med 2007;13:461-9.

[17] Hulscher MEJL, Wensing M, Van Der Weijden T, Grol R. Interventions to implement prevention in primary care (Review). 2005.

[18] Sanchez A, Grandes G, Cortada JM, Pombo H, Balague L, Calderon C. Modelling innovative interventions for optimising healthy lifestyle promotion in primary health care : " Prescribe Vida Saludable" phase I research protocol. BMC Health Serv Res 2009;10:1-10.

[19] Wensing M, Wollersheim H, Grol R. Organizational interventions to implement improvements in patient care : a structured review of reviews. Implement Sci 2006;9:19.

[20] National Board of Health and Welfare. National Guidelines for health promotion and disease prevention 2011. 2011.

[21] Scott JG, Cohen D, DiCicco-Bloom B, Orzano a J, Gregory P, Flocke S a, et al. Speaking of weight: how patients and primary care clinicians initiate weight loss counseling. Prev Med (Baltim) 2004;38:819-27.

[22] Flocke S a, Kelly R, Highland J. Initiation of health behavior discussions during primary care outpatient visits. Patient Educ Couns 2009;75:214-9.

[23] Pilnick A, Coleman T. 'I'll give up smoking when you get me better": patients' resistance to attempts to problematise smoking in general practice (GP) consultations. Soc Sci Med 2003;57:135-45.

[24] Jallinoja P, Absetz P, Kuronen R, Nissinen A, Talja M, Uutela A, et al. The dilemma of patient responsibility for lifestyle change : Perceptions among primary care physicians and nurses. Scand J Prim Heal Careealth Care 2007;25:244-9.

[25] Parker W, Steyn NP, Levitt NS, Lombard CJ. Health promotion services for patients having non-comminicable diseases: feedback from patients and health care providers in Cape Town, South Africa. BMC Public Health 2012;12:503.

[26] Glaser B. Doing Grounded Theory: Issues and discussions. Sociology Press; 1998.

[27] Ryan RM, Deci EL. Self-determination theory and the facilitation of intrinsic motivation, social development, and well-being. Am Psychol 2000;55:68-78.

[28] Michie S, van Stralen MM, West R. The behaviour change wheel: a new method for characterising and designing behaviour change interventions. Implement Sci 2011;6:42.

[29] Vermeire E, Hearnshaw H, Van Royen P, Denekens J. Patient adherence to treatment: three decades of research. A comprehensive review. J Clin Pharm Ther 2001;26:331-42.

[30] Chatterjee JS. From compliance to concordance in diabetes. J Med Ethics 2006;32:50710. 
[31] Proctor E, Silmere H, Raghavan R, Hovmand P, Aarons G, Bunger A, et al. Outcomes for implementation research: conceptual distinctions, measurement challenges, and research agenda. Adm Policy Ment Health 2011;38:65-76.

[32] Walseth LIVT, Abildsnes E, Schei E. Patients ' experiences with lifestyle counselling in general practice: A qualitative study. Scand J Prim Heal Care 2011;29:99-103.

[33] Street RL, Makoul G, Arora NK, Epstein RM. How does communication heal? Pathways linking clinician-patient communication to health outcomes. Patient Educ Couns 2009;74:295-301.

[34] Holmström I, Röing M. The relation between patient-centeredness and patient empowerment: a discussion on concepts. Patient Educ Couns 2010;79:167-72.

[35] Cohen DJ, McDaniel RR, Crabtree BF, Ruhe MC, Weyer SM, Tallia AF, et al. Cohen 2004 A practice change model for QL improvement in PC practice. J Healthc Manag 2004;49:155-68.

[36] Isaacson N, Holtrop JS, Cohen D, Ferrer RL, McKee MD. Examining role change in primary care practice. J Prim Care Community Health 2012;3:195-200. 
Figure 1: Substantive theory of being healthy. Core category: being healthy and interconnected categories: conditions for being healthy, managing being healthy and interactions about being healthy.

Fig. 2. Patient trajectories for being healthy for each patient type. 
Table 1. Patient characteristics: age, gender, employment, and education.

\begin{tabular}{lc}
\hline & No. \\
\hline Age & 3 \\
30-39 years & 3 \\
40-49 years & 5 \\
50-59 years & 7 \\
60-69 years & 4 \\
70+ years & \\
Gender & 15 \\
Women & 7 \\
Men & \\
Education & \\
No formal education & 4 \\
Year 9 & 10 \\
Year 12 & 8 \\
University degree & \\
Employment & 1 \\
Student & 15 \\
Employed & 6 \\
Retired & \\
\hline
\end{tabular}


Figure 1: Substantive theory of being healthy. Core category: being healthy and interconnected categories: conditions for being healthy, managing being healthy and interactions about being healthy.

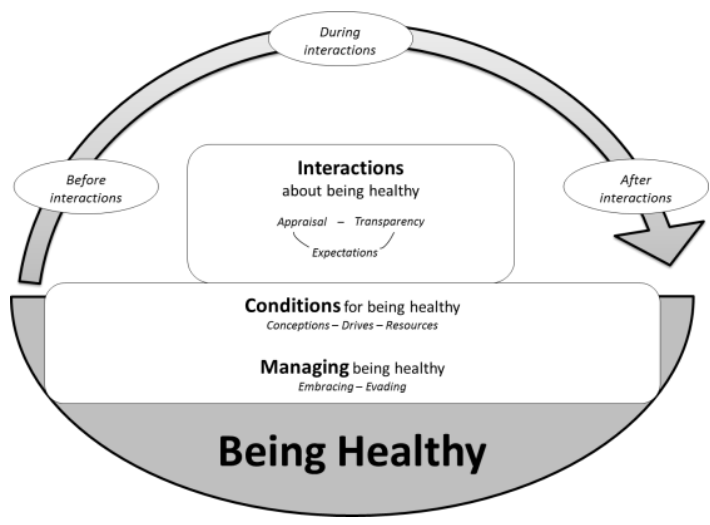


Figure 2: Patient trajectories of being healthy for each patient type.

A: RESIGNED

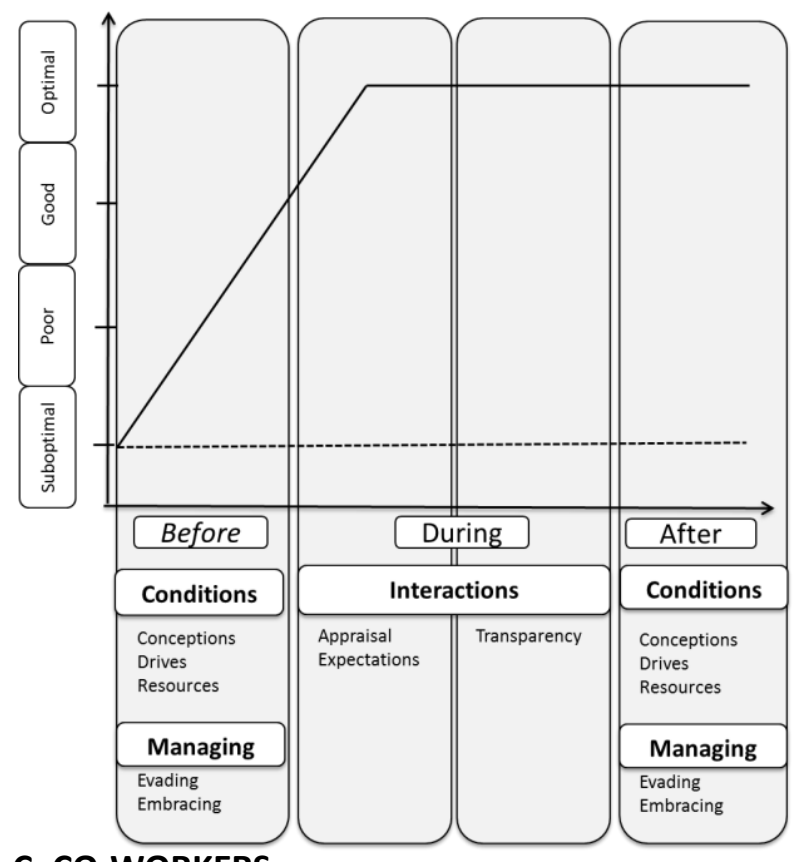

C: CO-WORKERS

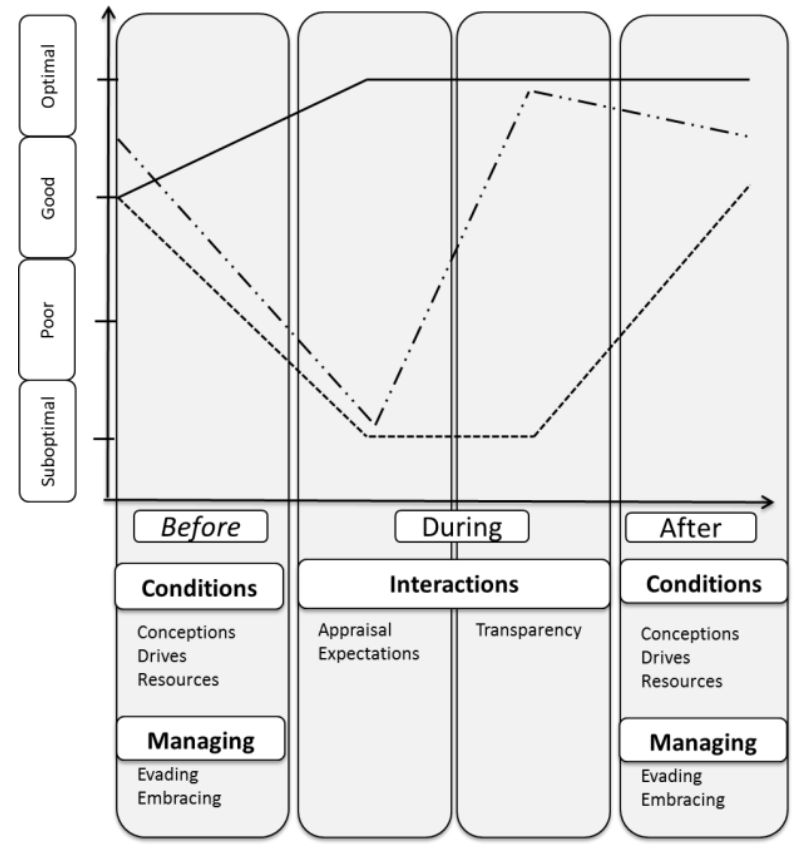

B: RECEIVERS

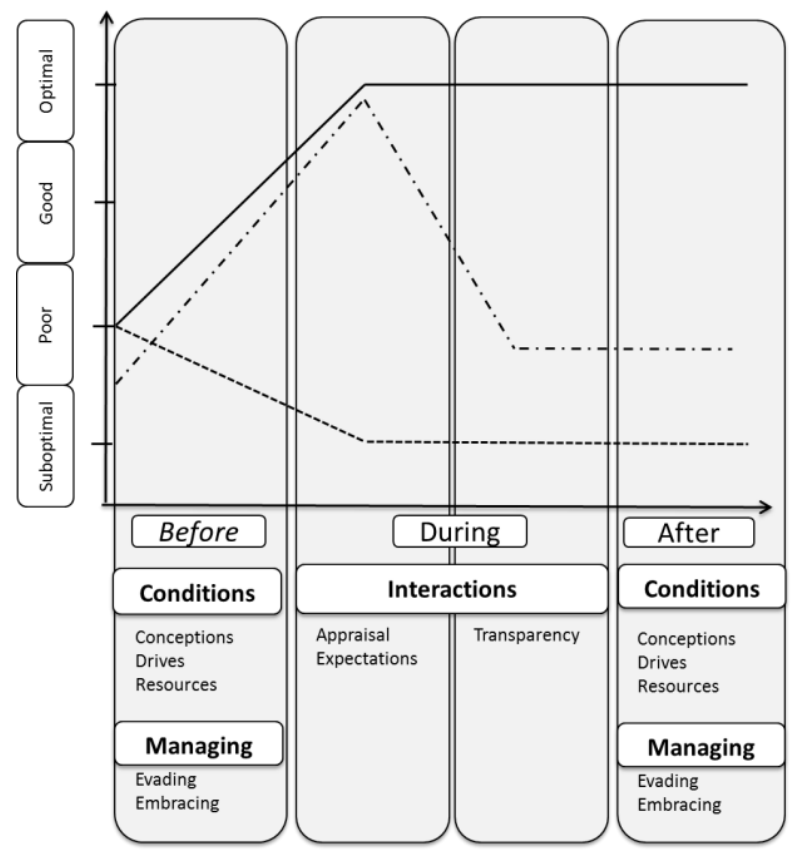

D: LEADERS

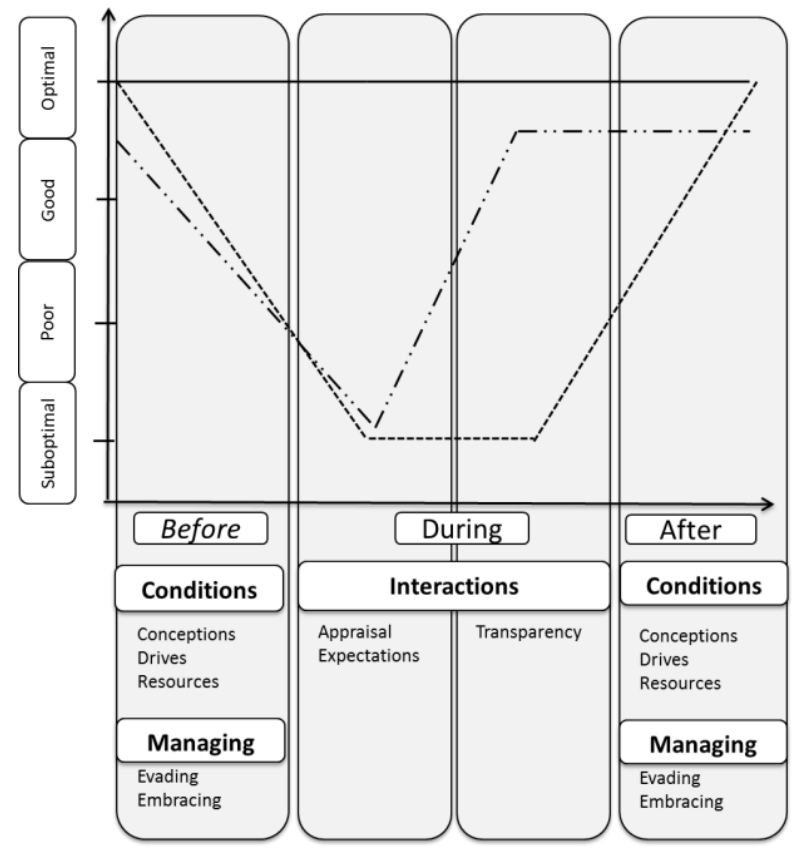

Positive situation appraisal

---- Negative situation appraisal

-.... Positive situation appraisal but limited internal drive

- . Negative situation apprisal but expectations of an active patient role 
